\title{
Analysis of Network Throughput and Link Failure Impact in Photonic Switched Metro-Access Networks
}

\author{
Indayara B. Martins, F. Rudge Barbosa, L. H.Bonani, E. Moschim
}

\begin{abstract}
Here we investigate the performance of optical packet/burst switched (OPS/OBS) architectures connected as mesh and as ring topologies, to be applied in future optical metropolitan networks, bringing more transparency to these networks. Network throughput and protection to link failure under uniform traffic distribution is investigated in order to evaluate the sensitivity of OPS/OBSN performance. Our results are based on analysis of simulations and comparison between various mesh (Manhattan St. type) and ring topologies. We also consider the traffic distributions over the network links and the impact caused by failure of different links, thus providing a way to select protection deployment.
\end{abstract}

Index Terms- photonic switching, network protection, Optical Fiber Communications.

\section{INTRODUCTION}

Whereas core networks deal with highly aggregated traffic, the edge (access and distribution) networks require granularity and flexibility to deal with highly variable traffic. For core optical networks, WDM solutions are well established. However, at the metropolitan access level, a tendency to use optical packets for transport and switching following today's (electronic) packet-based optical networks environment are being proposed and tested [1]-[5], carrying high bandwidths and running over single wavelengths.

In the present work we propose and analyze the dynamic behavior of optical packet/burst switching (OPS/OBS) networks based mainly on mesh topologies designed for application at the metropolitan access level. Due to the success of ring topology, widely used in present day SDH/SONET optical networks, we include in our modeling and analysis a comparison with ring topologies, in terms of capacity and resilience of the network to transported traffic. The present OPS/OBS asynchronous solution is original to the best of our knowledge [1][2][3], and does not require OE conversion at optical nodes, with switching and routing being performed directly at the optical layer level. The present work improves on previous results [2][3], by presenting better methods for throughput evaluation and a system to evaluate critical link failure in mesh and ring topologies. Network performance is analyzed according to common parameters, such as average number of hops, distribution of link occupation, and network throughput. Protection of traffic in these multi-hop networks is an important aspect to be considered [2][7][10], and our

Authors are with the School of Electrical and Computer Engineering, University of Campinas - FEEC-Unicamp, Campinas-SP - Brazil, \{ibertold, rudge,moschim\}@dsif.fee.unicamp.br; tel +55-19-3521-3766, and Federal University of ABC, UFABC, S.AndréSP, Brazil, \{luiz.bonani@ufabc.edu.br\} analysis includes the impact of link failure on these OPS/OBS networks. To the best of our knowledge this is the first time that protection of OPS/OBS networks in both ring and mesh topologies is considered in a direct comparison. The better application of these networks is in the metro-access network range, implemented on single wavelength. For larger distances or higher capacity demands a WDM network can be considered [5][6][9], but not in the scope of this work.

\section{Optical Packet Switching Network and Topologies}

The OPS/OBS network architecture in Fig. 1 is a Manhattan St. type topology, consisting of $2 \times 2$ optical network nodes, interconnected unidirectionally, and having optical add-drop functionalities. The indicated higher node allows connection to other sub-networks in a WDM environment, with details given in another work [5].

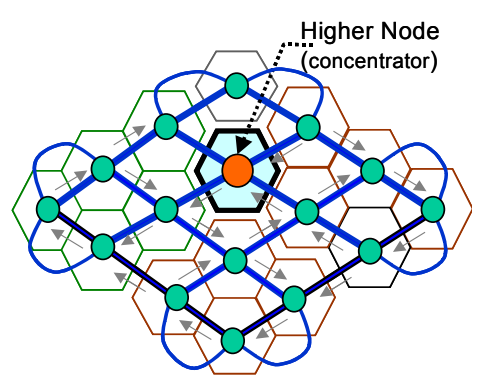

Fig. 1. Unidirectional Mesh topology optical network.

The optical switching is controlled by fast electronic logic circuits (40ns rise-fall times) operating on packet-by-packet basis, determined only by processing the header. The overall switching time is $<2 \mu$ s for every packet or burst, independent of payload size. This simplified architecture allows the use of deflection routing (DR) as the protocol to resolve contentions and avoid collisions [3][4], with the advantage of no need for buffering in the optical layer, which contributes to cost reduction. Electronic buffering at the (external) client interface of the optical node is considered elsewhere [5], and does not affect the results of the present work. The optical node dynamics is the following. An optical packet/burst arriving at the optical node blocks the switch to its preferred route; another packet/burst arriving within the same blocking duration will be deflected to the available outport. This simplifies the optical nodes, allowing the network to be operated asynchronously, with packets/bursts guided only by their own header [3]. We believe that our solution is best suited for metropolitan access area, with links having typically $<10 \mathrm{~km}$ length, and networks spanning less than 36 nodes. 
Mesh and ring topologies have been chosen for the analysis of traffic throughput and to evaluate the degree of use (load) of each unidirectional link (bi-directional networks require different boundary conditions and will be considered in future work).

\section{BASIC THEORY AND PARAMETERS}

The total capacity of the network is defined as [3][4],

$$
C_{t}=\frac{2 \cdot N \cdot S}{\bar{H}}
$$

where, $H$ is the total average number of hops for the optical packets (from origin to destination), $N$ number of nodes and $S$ link capacity; the factor 2 comes from the two possible output paths at every node. We assume Poisson distribution, with every node generating uniform traffic (number of applications) to every other node except itself, and unidirectional links. The effective number of users in the network for this condition is $N_{u}=N(N-1)$. We define the user-share capacity as $C t / N u$, which using (1) becomes,

$$
C_{u}=\frac{2 . S}{\bar{H} \cdot(N-1)}
$$

A figure of merit for network performance is defined as $F_{p}$ $=C t / H$, which can be applied to any multihop environment that follows (1). The rationale is simple and useful: higher capacity with smaller average hop number leads to a more efficient network that will also have lower latency (considering as we did, links of same size).

In the case of link failure, it is expected that network capacity decreases because of the lost link. The number of possible paths will decrease, and equation (1) should be changed. In equation (1), $2 N$ is the total number of links, if $m$ links fail they must be subtracted, and equation (1) becomes,

$$
C_{t}=\frac{(2 N-m) \cdot S}{\bar{H}}
$$

We will be considering only single link failure, $m=1$, because two or more simultaneous failures may occur but present very low probability $\left(<10^{-5}\right)[2]$.

For deflection routing (DR) protocol, the throughput $T_{p}$ is evaluated considering the average number hops $H$ for each value of network load $L_{c}$, because path availability is dependent on load, and will vary inversely with it (higher load will increase $H$ and from (1), capacity will tend to saturate). $L_{c}$ varies in $[0,1]$ interval.

The network throughput is given by [6] :

$$
T_{p}=C_{t} \cdot L_{c} .
$$

In a previous work [2] we had considered only one method to evaluate the average number of hops from origin to destination for both regular and quasi-regular networks. However, we have noticed that for regular MS-type (even number of rows and columns) networks and DR protocol we can find the throughput by considering any single destination node and then generalize for all nodes, because for a regular network the throughput results the same for all destination nodes. On the other hand, for quasi-regular MS-type (designated MSq, with odd number of rows and columns) networks it is necessary to find the throughput for each destination node and afterwards take an average. Therefore, a general expression for $T p$ can be written as

$$
T_{P}=\frac{\sum_{i=1}^{N} T_{p i}}{N}
$$

where $i$ is the destination node, $\mathrm{T}_{\mathrm{pi}}$ the partial throughput to that node, and $N$ the total number of nodes in the network.

\section{Simulation Methods and Network Configurations}

Here we summarize the procedures and conditions for this work. We have chosen, as defined above, the MS-type mesh topologies MSq-9, MS-16, MSq- 25, and ring topologies with 9, 16, 25 nodes. Link transmission bit-rate is $2.5 \mathrm{~Gb} / \mathrm{s}$, and link length $10 \mathrm{~km}$.

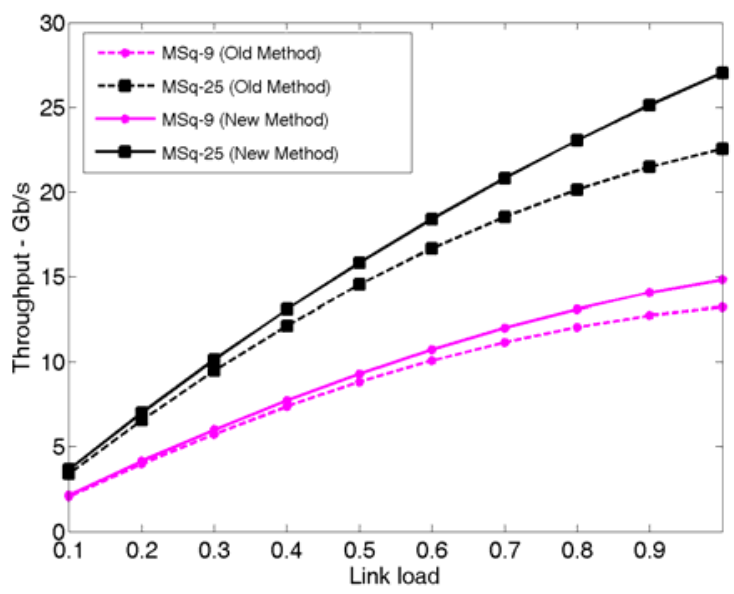

Fig. 2 - Comparative Throughput for mesh networks using old and new methods (see text).

All links are unidirectional and traffic distribution is assumed as uniform (every node generates the same traffic to every other node). Applications are defined as the total of optical packets transmitted from a given node to all other nodes, and the sum of all applications is the total traffic load on the network. For protection analysis we consider only single link failure [2][8][9].

The results for OPS/OBS network traffic simulations were obtained using the software Network Simulator (NS-2); for analytic results, data processing and plotting the MatLab ${ }^{\circledR}$ was used.

\section{NETwOrk RESUltS AND DiscUSSION}

Results of throughput for regular and quasi-regular mesh topologies and ring topologies are shown in Fig. 3. We can see that the performance of OPS/OBSN mesh networks is 
markedly superior to ring topology, especially in higher node counts. Some features can be noted in this MS-type network model: the 4-node ring is the same as the 4-node "mesh"; the 16-node mesh in comparison with the 9-node almost doubles the node number but does not improve performance accordingly; the 25-node mesh more than doubles the network capacity and throughput as compared to the equivalent ring. These features clearly point to mesh topologies as more adequate to high capacity edge networks, not considering installation costs. We will see that failure analysis reinforces this observation.

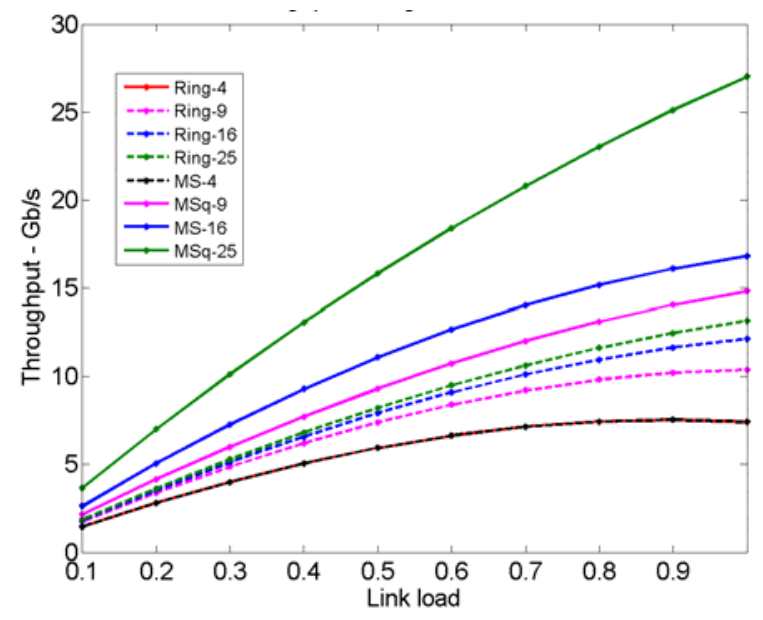

Fig. 3 - Comparative Throughput for mesh and ring networks using the new method.

\section{LINK FAILURE ANALYSIS}

With the above considerations in mind, one has proceeded to evaluate the impact of link failure on network throughput. The detailed analysis of traffic distribution is presented in another work [10]; here we use the more relevant results. For both networks the more used links obtained in [2] were chosen, and only one link is broken at a time. In the case of mesh network, the failure of less used links made no noticeable difference in network performance, whereas for ring networks the impact was practically the same as with the more used links.

Fig. 4 presents the comparative results for the throughput in the ring and mesh networks, with and without failure. For clarity, only the 16 -node is shown. The complete set of simulations include the MSq-9, the MS-16, and the MSq-25, as well as the corresponding rings (as in Fig.3).

We observe that the mesh topologies moderately reduce their performance, with respective throughput reduction averages of $11 \%, 6,5 \%, 4 \%$ (for load $L c=100 \%$ ). For the corresponding ring topologies the observed throughputs reduce as much as $26 \%, 25 \%, 25 \%$, respectively, demonstrating the fragility of ring as compared to mesh. Moreover, since all links are practically equivalent in ring, it is more difficult not only to decide where to protect (protection for the whole network is too costly), but also to locate failure and to provide restoration, considering that the modelled networks span for tens of $\mathrm{km}$. In the mesh topologies these difficulties are minimized [7] [10].

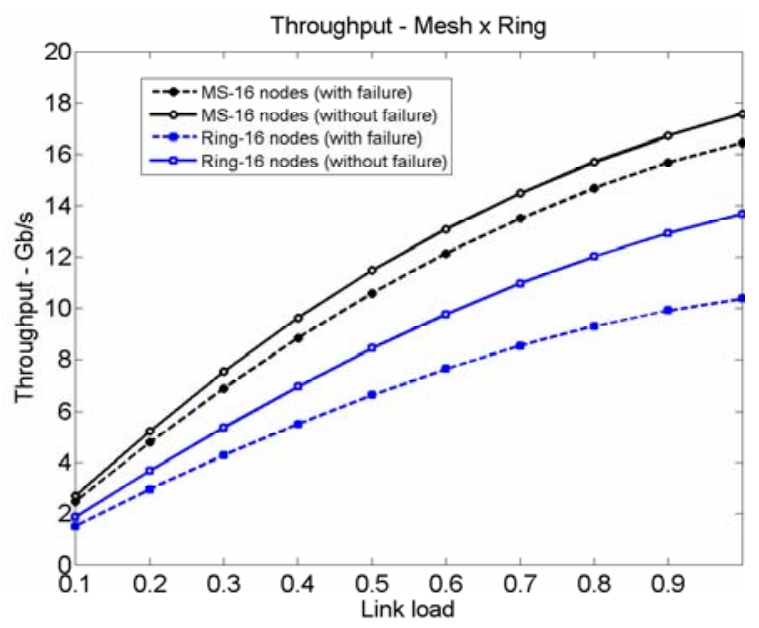

Fig. 4 - Network Throughput for MS-16 and Ring-16 (with and without failure).

\section{CONCLUSION}

Following the MS-type network model for regular and quasi-regular networks, we have updated and improved our results on multi-hop traffic simulation for OPS/OBS networks.

We have also performed comparative analysis of capacity and throughput for mesh and ring topologies, confirming the superior performance of the mesh networks. Detailed installation costs are out of the scope of this work, but it should be noted that our model is based on asynchronous operation of optical packet switching and routing node, where packets are routed and processed only by their headers; thus a simplified bufferless optical architecture that uses deflection routing (DR), seems to constitute a set of relaxed requirements in the direction of cost reduction with advanced performance.

Concerning network protection, we have accordingly verified with simulation results that link failure has a more significant impact on rings than in equivalent mesh topologies, indicating a superior performance of mesh topology. This is an important difference between the two topologies that this work highlights, because in mesh different paths imply different loads on links, whereas in ring all links have practically the same requirement of protection, and decisions prior to installation can be more difficult. Results show that impact of failures is less critical in reducing capacity of mesh networks than of equivalent ring topologies, confirming that mesh networks are more robust with higher service survivability.

Finally, we expect that the present work contributes to better understanding of optical traffic dynamics in future OPS/OBS networks and to identify which network topology connections are more critical to be protected and rapidly restored.

\section{REFERENCES}

[1] S. J. Yoo, "Optical Packet and Burst Switching Technologies for the Future Photonic Internet", J. Lightwave Technology, vol. 24, no. 12, december 2006. 
[2] I. B. Martins, L. H. Bonani, E. Moschim, F. Rudge Barbosa, "Comparison of Link failure and Protection in Ring and Mesh OPS/OBS Metropolitan Area Optical Networks", Proc 13th Symp. on Microwave and Optoelectronics-MOMAG'2008, Sept. 2008, Floripa SC, Brazil.

[3] I. B. Martins, L. H. Bonani, F. R. Barbosa, E. Moschim, "Dynamic Traffic Analysis of Metro Access Optical Packet Switching Networks having Mesh Topologies", Proc. Int. Telecom Symp., ITS'2006, Sept. 2006, Fortaleza, Brazil.

[4] S. Yao, B. Mukherjee, S. J. Yoo, S. Dixit, "A Unified Study of Contention Resolution Schemes in Optical packet switching Networks", IEEE J. Lightwave Tech, vol.21, no.3, p.672, March 2003.

[5] L.H. Bonani, F. Rudge Barbosa, E. Moschim, R. Arthur "Analysis of Eletronic Buffers in Optical Packet/Burst Switched Mesh Networks", International Conference on Transport Optical Networks-ICTON-2008, June 2008 - Athens, Greece.

[6] R. Ramaswami, K.N. Sivarajan, Optical Networks: a practical perspective, Morgan Kaufmann Publishers, $2^{\text {nd }}$ Edition, 2002.

[7] M. Held, L. Zhou, "Redundancy, Restorability and Path Availability in Optical Mesh Networks", International Conference on Transparent Optical Networks - ICTON, June - 2006, Nottingham, United Kingdom.

[8] T. Cinkler, L. Gyarmati, "Mpp: Optimal Multi-Path Routing with Protection", proceeding Int. Conf. Communications-ICC-2008- Beijing, China.

[9] D. A. Schupke and R. Prinz. "Capacity, Efficiency and Restorability of Path Protection and Rerouting in WDM Networks Subject to Dual Failures", Photonic Network Comm., Vol 8, n. 2, p.191, Springer, Netherlands, Sept. 2004.

[10] I.B.Martins, LHBonani, FRBarbosa, E.Moschim, "Improved Method for Evaluation of Network Throughput na Protection in Future Optically Switched Metropolitan Networks", Proc. Fifth Advanced International Conference on Telecommunications - AICT'2009, May 2009, Venice, Italy. 